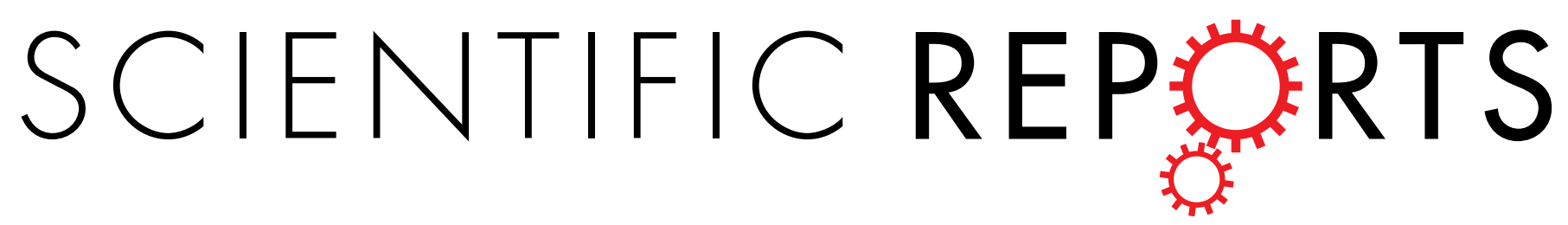

OPEN Publisher Correction: Inferring

Published online: 14 March 2018

\title{
structural connectivity using Ising couplings in models of neuronal networks
}

Balasundaram Kadirvelu, Yoshikatsu Hayashi \& Slawomir J. Nasuto

Correction to: Scientific Reports https://doi.org/10.1038/s41598-017-05462-2, published online 15 August 2017

The HTML version of this Article contains an error in the order of the Figures. Figures 1, 2 and 3 were published as Figures 2, 3 and 1 respectively. The correct Figures appear below. The Figure legends are correct. The PDF version was correct at the time of publication.

Brain Embodiment Lab, Biomedical Engineering, School of Biological Sciences, University of Reading, Reading, United Kingdom. Correspondence and requests for materials should be addressed to B.K. (email: B.Kadirvelu@pgr. reading.ac.uk) 

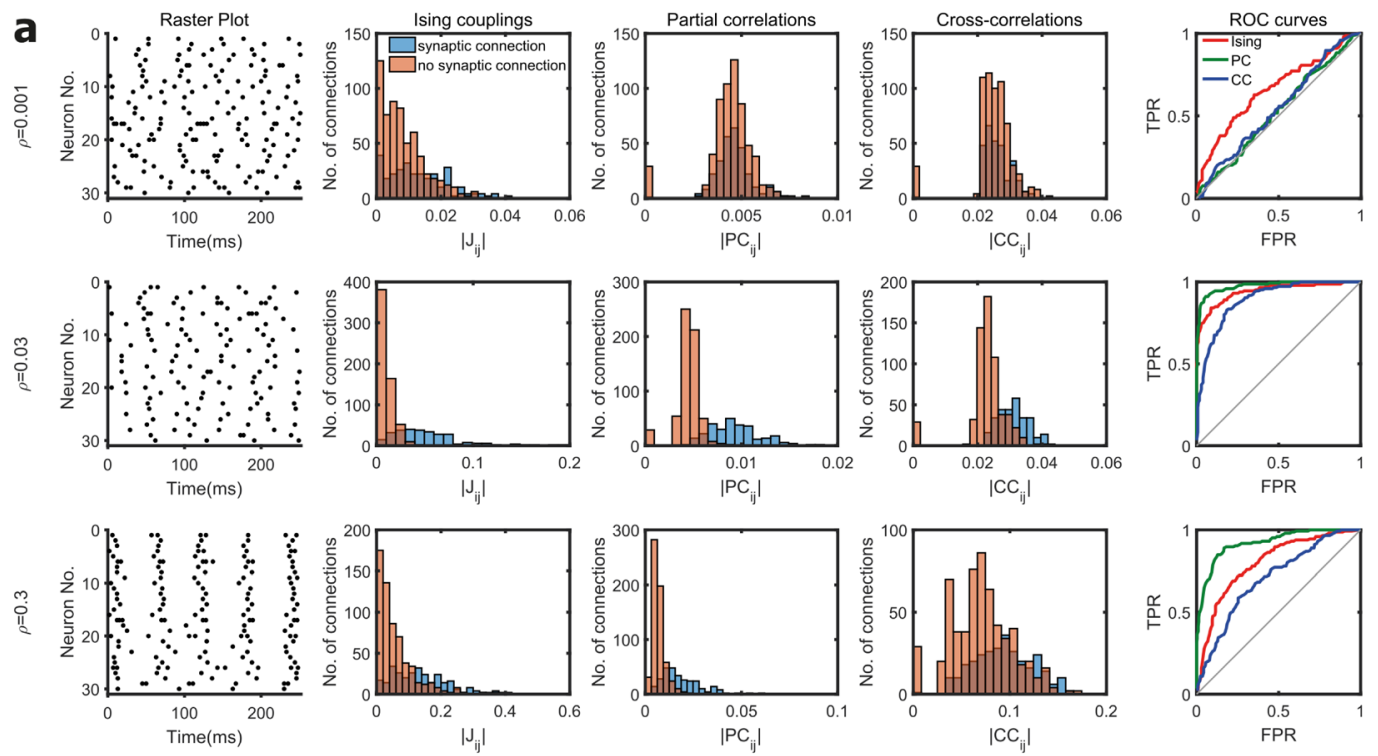

b

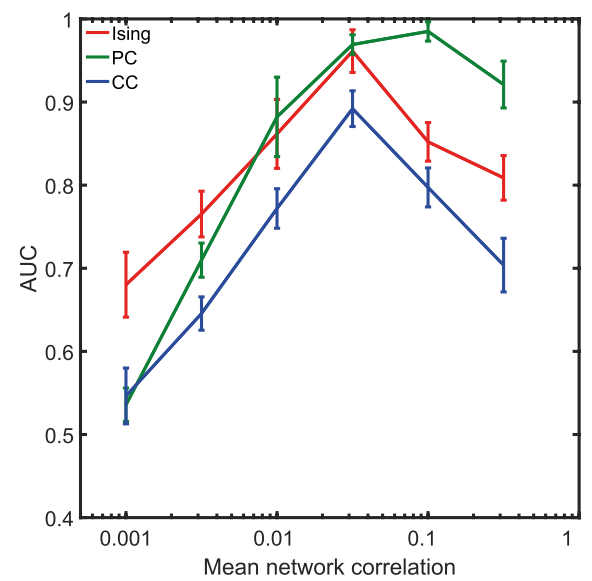

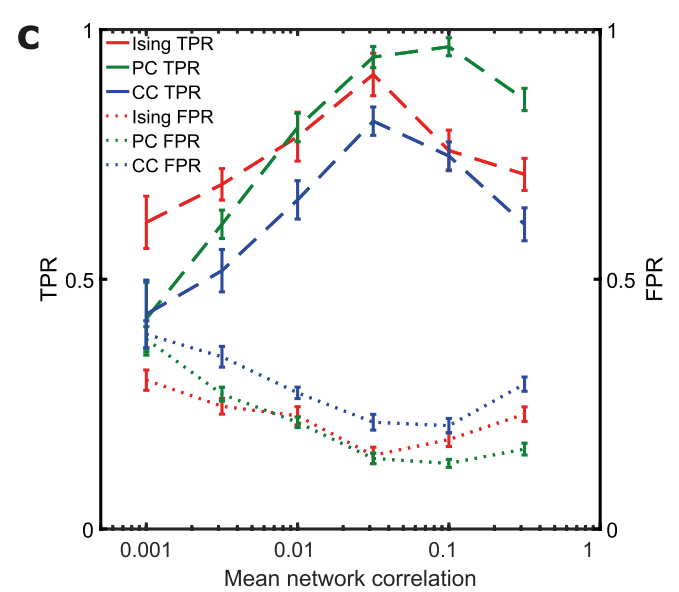

Figure 1. Effect of mean network correlation. (a) The first column in each row shows the raster plot of the spiking activity from a simulated neuronal network for a firing rate of $20 \mathrm{~Hz}$ and different network correlation levels. Histogram of the Ising couplings, partial correlations and cross-correlations for the pairs of neurons that are synaptically connected and not connected are shown respectively in the second, third and fourth columns. The corresponding ROC curves of the three functional connectivity metrics are shown in the last column. The first, second and third rows correspond to mean network correlation levels $(\rho)$ of $0.001,0.03$ and 0.3 respectively. (b) Plot of the AUC values for different mean network correlation levels in scale-free networks of 30 neurons for a fixed firing rate of $20 \mathrm{~Hz}$. Mean value was calculated from ten simulated networks. For weaker correlation levels (0.001 and 0.003$)$, AUC value of Ising couplings was significantly higher than partial and cross-correlations. For stronger correlation levels ( 0.1 and 0.3$)$, partial correlations had a significantly higher AUC value compared to Ising couplings and cross-correlations ( $p<0.01$, two-sample t-tests). (c) True positive rate (TPR) and false positive rate (FPR) for the reconstruction of the structural connections by the three functional connectivity metrics thresholded at a sparsity threshold value of $20 \%$. 

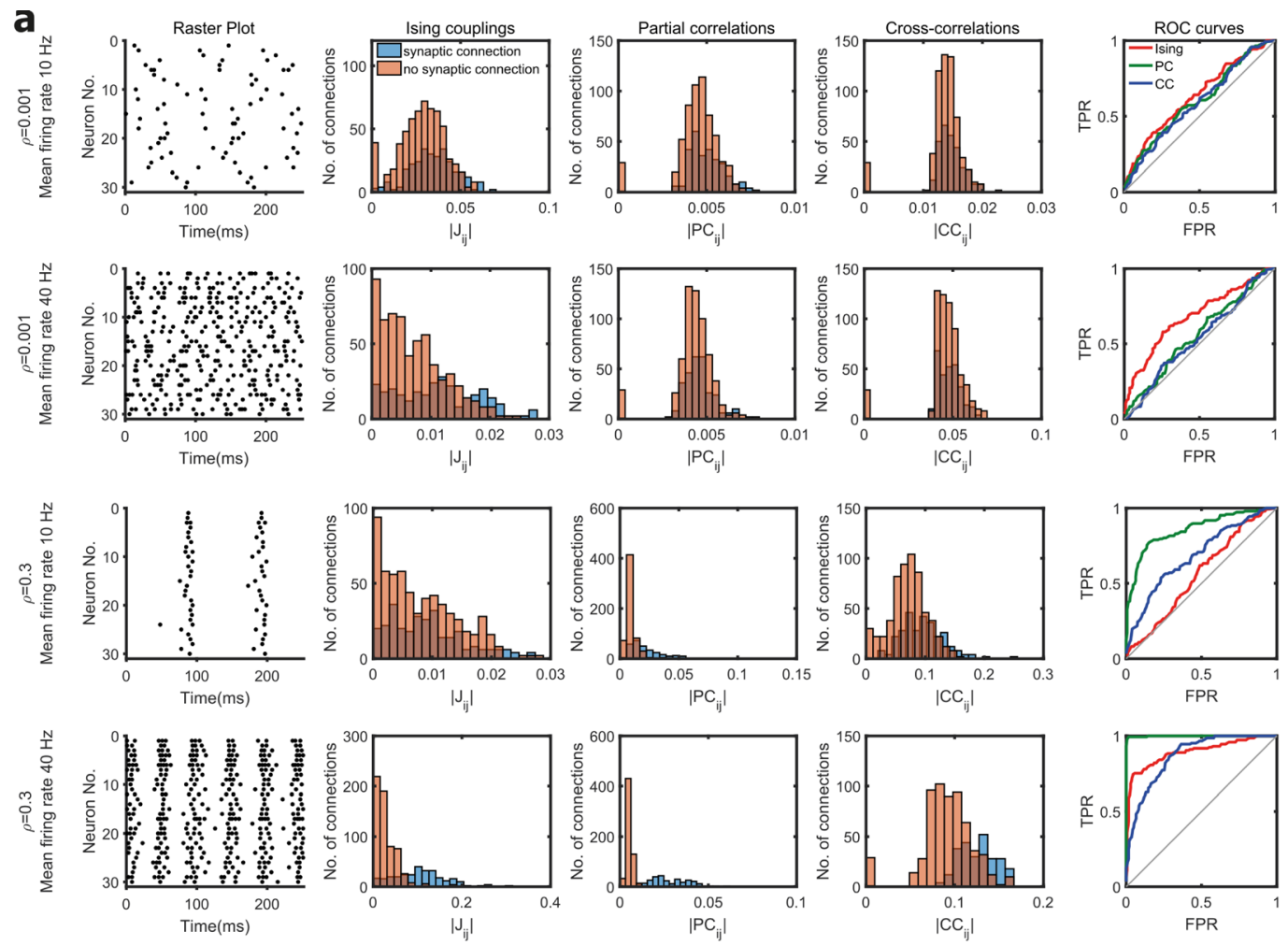

b

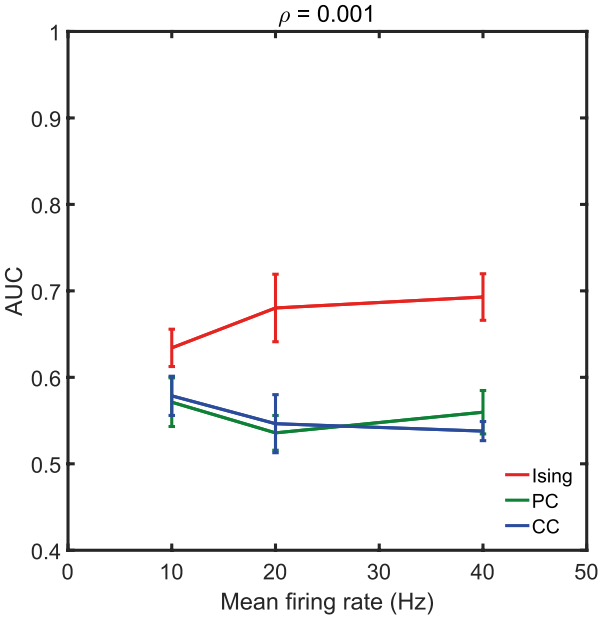

C

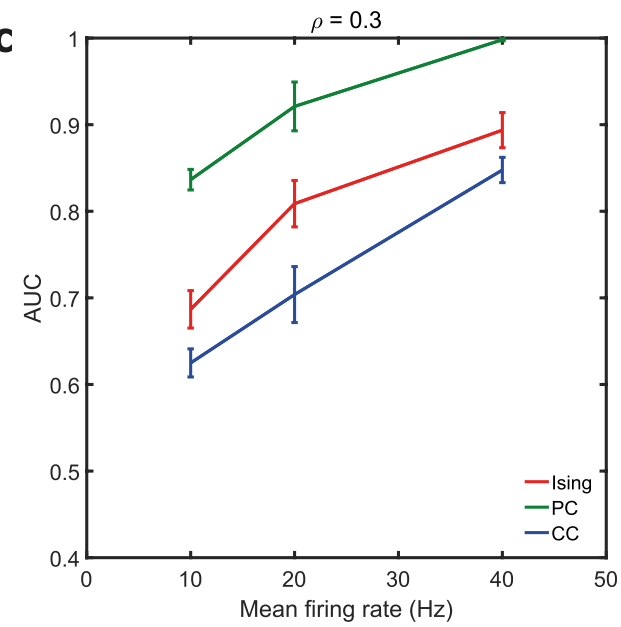

Figure 2. Effect of mean network firing rate. (a) The first and second rows correspond to firing rates of $10 \mathrm{~Hz}$ and $40 \mathrm{~Hz}$ respectively for a fixed correlation level $(\rho)$ of 0.001 . The third and fourth rows correspond to firing rates of $10 \mathrm{~Hz}$ and $40 \mathrm{~Hz}$ respectively for a fixed correlation level of 0.3 . Raster plot of the spiking activity from a simulated neuronal network is shown in the first column. Histogram of the Ising couplings, partial correlations and cross-correlations for the pairs of neurons that are synaptically connected and not connected are shown respectively in the second, third and fourth columns. The corresponding ROC curves of the three functional connectivity metrics are shown in the last column. (b) and (c) Plot of the AUC values for different firing rates in scale-free networks of 30 neurons for fixed mean network correlation levels of 0.001 and 0.3 respectively. Mean value was calculated from ten simulated networks. 

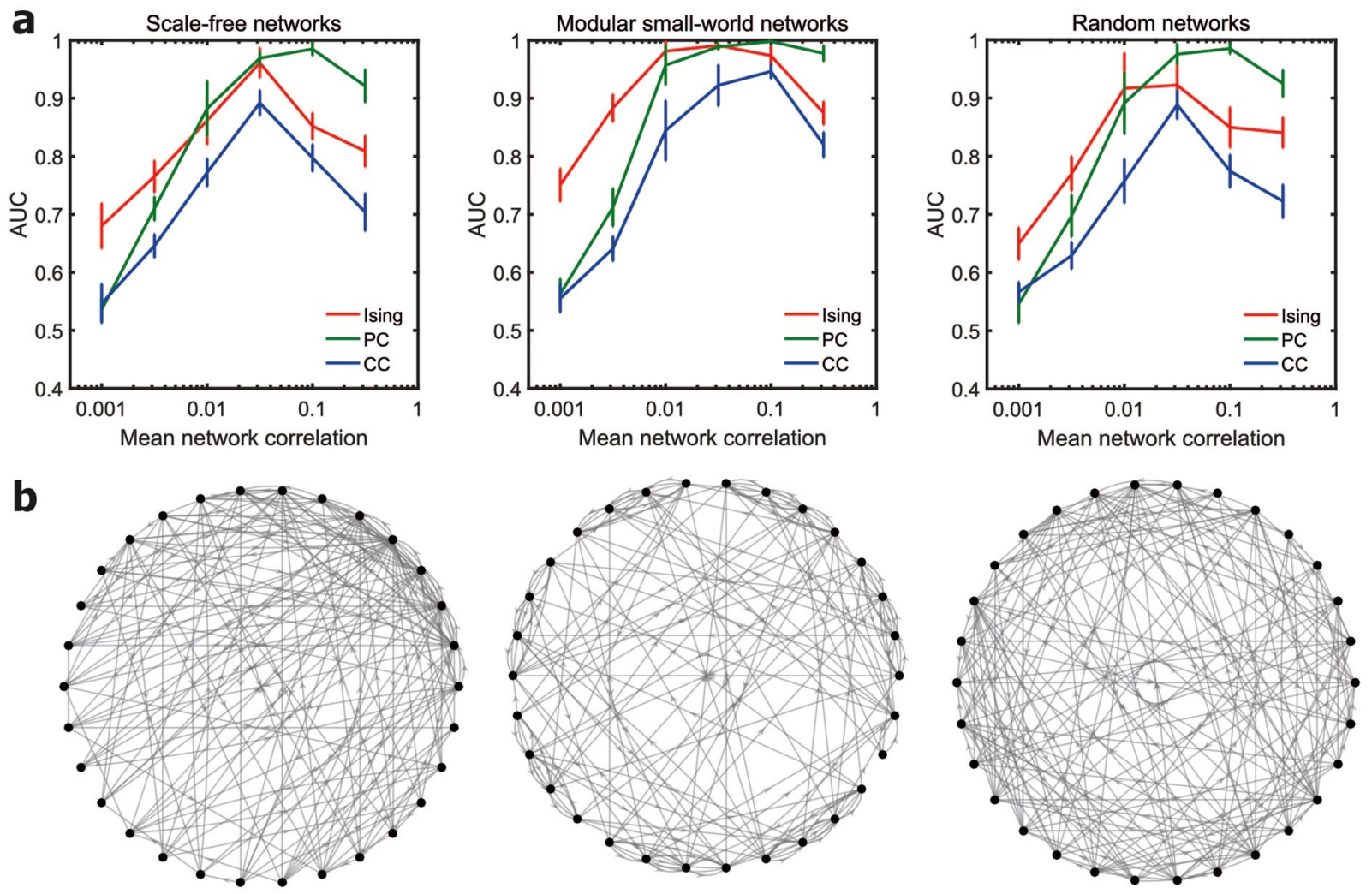

Figure 3. Effect of network topology. (a) Plot of the AUC values for networks of 30 neurons with scalefree (SF), small-world (SW) and Erdos-Renyi (ER) random network topologies. Data was averaged over ten simulated networks for each network condition. Firing rate was fixed at $20 \mathrm{~Hz}$ in all cases. All the three topologies had the same link density of 0.2. (b) An example of the structural connectivity network for each topology. Scale-free networks form a few highly connected hub nodes. Modular small-world networks present a balance of segregation and integration via dense intra-module connections and sparse inter-module connections. Most nodes in random networks have a degree in the vicinity of the average degree of the network.

(c) Open Access This article is licensed under a Creative Commons Attribution 4.0 International (c) License, which permits use, sharing, adaptation, distribution and reproduction in any medium or format, as long as you give appropriate credit to the original author(s) and the source, provide a link to the Creative Commons license, and indicate if changes were made. The images or other third party material in this article are included in the article's Creative Commons license, unless indicated otherwise in a credit line to the material. If material is not included in the article's Creative Commons license and your intended use is not permitted by statutory regulation or exceeds the permitted use, you will need to obtain permission directly from the copyright holder. To view a copy of this license, visit http://creativecommons.org/licenses/by/4.0/.

(c) The Author(s) 2018 